\title{
Integrality Gap of the Hypergraphic Relaxation of Steiner Trees: a short proof of a 1.55 upper bound
}

\author{
Deeparnab Chakrabarty Jochen Könemann David Pritchard
}

October 23, 2018

\begin{abstract}
Recently, Byrka et al. [1] gave a 1.39-approximation for the Steiner tree problem, using a hypergraph-based LP relaxation. They also upper-bounded its integrality gap by 1.55 . We describe a shorter proof of the same integrality gap bound, by applying some of their techniques to a randomized loss-contracting algorithm.
\end{abstract}

\section{Introduction}

In the Steiner tree problem, we are given an undirected graph $G=(V, E)$ with $\operatorname{costs} c$ on edges and its vertex set partitioned into terminals (denoted $R \subset V$ ) and Steiner vertices $(V \backslash R)$. A Steiner tree is a tree spanning all of $R$ plus any subset of $V \backslash R$, and the problem is to find a minimum-cost such tree. The Steiner tree problem is APX-hard, thus the best we can hope for is a constant-factor approximation algorithm.

The best known ratio is a result of Byrka, Grandoni, Rothvoß and Sanità [1]: their randomized iterated rounding algorithm gives approximation ratio $\ln (4)+\epsilon \approx 1.39$. The prior best was a $1+\frac{\ln 3}{2}+\epsilon \approx 1.55$ ratio, via the deterministic loss-contracting algorithm of Robins and Zelikovsky [6]. The algorithm of [1] differs from previous work in that it uses a linear programming (LP) relaxation; the LP is based on hypergraphs, and it has several different-looking but equivalent [2,5] nice formulations. A second result of [1] concerns the LP's integrality gap, which is defined as the worst-case ratio (max over all instances) of the optimal Steiner tree cost to the LP's optimal value. Byrka et al. show the integrality gap is at most 1.55, and their proof builds on the analysis of [6]. In this note we give a shorter proof of the same bound using a simple LP-rounding algorithm.

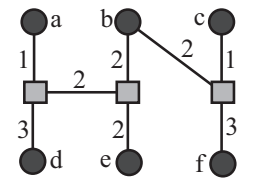

(i)

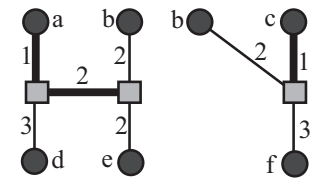

(ii)

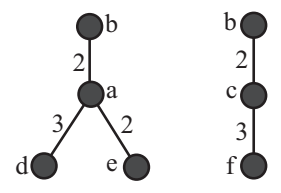

(iii)

Figure 1: In (i) we show a Steiner tree; circles are terminals and squares are Steiner nodes. In (ii) we show its decomposition into full components, and their losses in bold. In (iii) we show the full components after loss contraction. 
We now describe one formulation for the hypergraphic LP. Given a set $K \subset R$ of terminals, a full component on $K$ is a tree whose leaf set is $K$ and whose internal nodes are Steiner vertices. Every Steiner tree decomposes in a unique edge-disjoint way into full components; Figure 1(i) shows an example. Moreover, one can show that a set of full components on sets $\left(K_{1}, \ldots, K_{r}\right)$ forms a Steiner tree if and only if the hypergraph $\left(V,\left(K_{1}, \ldots, K_{r}\right)\right)$ is a hyper-spanning tree. Let $\mathrm{F}(K)$ denote a minimum-cost full component for terminal set $K \subset R$, and let $C_{K}$ be its cost. The hypergraphic LP is as follows:

$$
\begin{aligned}
\min & \sum_{K} C_{K} x_{K}: \\
\forall \varnothing \neq S \subseteq R: & \sum_{K: K \cap S \neq \varnothing} x_{K}(|K \cap S|-1) \leq|S|-1 \\
& \sum_{K} x_{K}(|K|-1)=|R|-1 \\
\forall K: \quad & x_{K} \geq 0
\end{aligned}
$$

The integral solutions of $(\mathcal{S})$ correspond to the full component sets of Steiner trees. As an aside, the $r$-restricted full component method (e.g. [4]) allows us to assume there are a polynomial number of full components while affecting the optimal Steiner tree cost by a $1+\epsilon$ factor. Then, it is possible to solve $(\mathcal{S})$ in polynomial time [1,8]. Here is our goal:

Theorem 1. [1] The integrality gap of the hypergraphic $L P(\mathcal{S})$ is at most $1+\ln 3 / 2 \approx 1.55$.

\section{Randomized Loss-Contracting Algorithm}

In this section we describe the algorithm. We introduce some terminology first. The loss of full component $\mathrm{F}(K)$, denoted by $\operatorname{Loss}(K)$, is a minimum-cost subset of $\mathrm{F}(K)$ 's edges that connects the Steiner vertices to the terminals. For example, Figure 1(ii) shows the loss of the two full components in bold. We let $\operatorname{loss}(K)$ denote the total cost of all edges in Loss $(K)$. The loss-contracted full component of $K$, denoted by $\operatorname{LC}(K)$, is obtained from $\mathrm{F}(K)$ by contracting its loss edges (see Figure 1(iii) for an example).

For clarity we make two observations. First, for each $K$ the edges of $\operatorname{LC}(K)$ correspond to the edges of $\mathrm{F}(K) \backslash \operatorname{Loss}(K)$. Second, for terminals $u, v$, there may be a $u v$ edge in several $\mathrm{LC}(K)$ 's but we think of them as distinct parallel edges.

Our randomized rounding algorithm, RLC, is shown below. We choose $M$ to have value at least $\sum_{K} x_{K}$ such that $t=M \ln 3$ is integral. $\operatorname{MST}(\cdot)$ denotes a minimum spanning tree and mst its cost. 


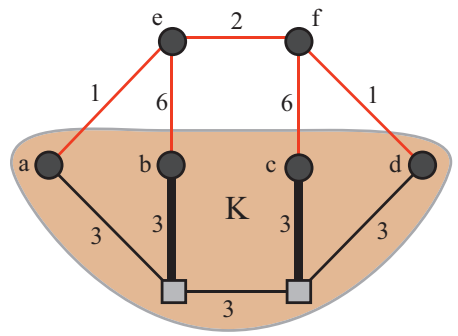

(i)

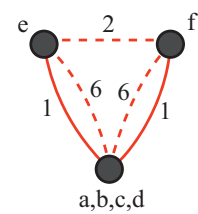

(ii)

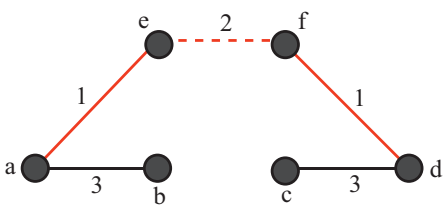

(iii)

Figure 2: In (i) we show a terminal spanning tree $T$ in red, and a full component spanning terminal set $K \subset\{a, b, c, d\}$ in black; thick edges are its loss. In (ii) we show $T / K$, and $\operatorname{Drop}_{T}(K)$ is shown as dashed edges. In (iii) we show $\operatorname{MST}(T \cup \operatorname{LC}(K))$.

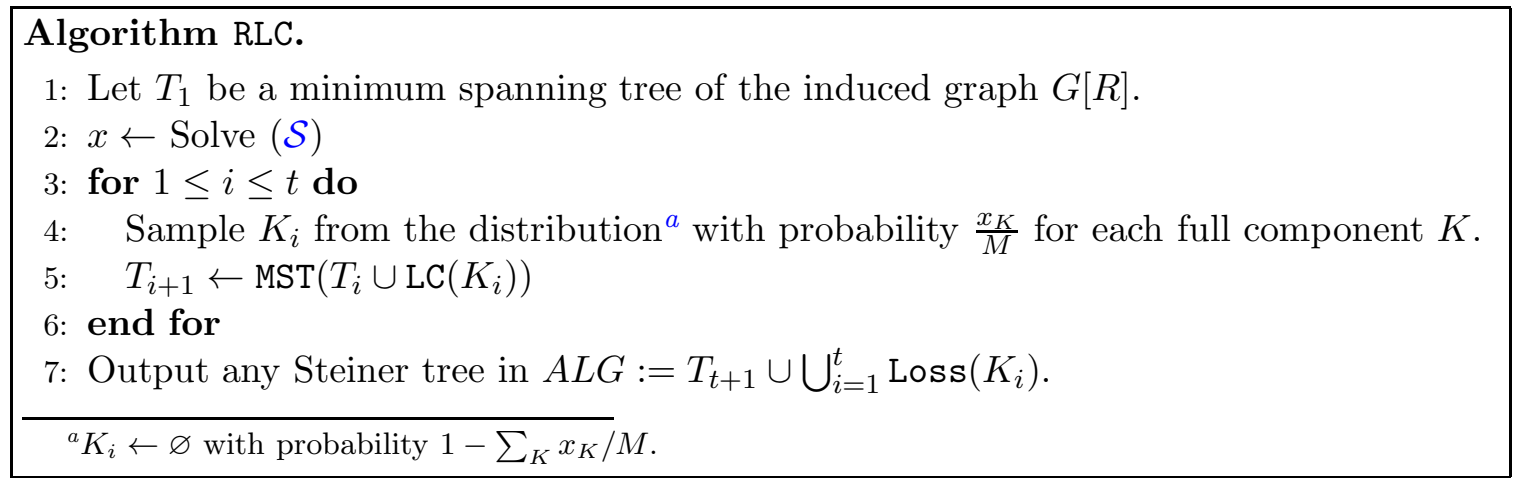

To prove that $A L G$ actually contains a Steiner tree, we must show all terminals are connected. To see this, note each edge $u v$ of $T_{t+1}$ is either a terminal-terminal edge of $G[R]$ in the input instance, or else $u v \in \operatorname{LC}\left(K_{i}\right)$ for some $i$ and therefore a $u$-v path is created when we add in $\operatorname{Loss}\left(K_{i}\right)$.

\section{Analysis}

In this section we prove that the tree's cost is at most $1+\frac{\ln 3}{2}$ times the optimum value of $(\mathcal{S})$. Each iteration of the main loop of algorithm RLC first samples a full component $K_{i}$ in step 4, and subsequently recomputes a minimum-cost spanning tree in the graph obtained from adding the loss-contracted part of $K_{i}$ to $T_{i}$. The new spanning tree $T_{i+1}$ is no more expensive than $T_{i}$; some of its edges are replaced by newly added edges in $\operatorname{LC}\left(K_{i}\right)$. Bounding the drop in cost will be the centerpiece of our analysis, and this step will in turn be facilitated by the elegant Bridge Lemma of Byrka et al. [1]. We describe this lemma first.

We first define the drop of a full component $K$ with respect to a terminal spanning tree $T$ (it is just a different name for the bridges of [1]). Let $T / K$ be the graph obtained from $T$ by identifying the terminals spanned by $K$. Then let

$$
\operatorname{Drop}_{T}(K):=E(T) \backslash E(\operatorname{MST}(T / K)),
$$


be the set of edges of $T$ that are not contained in a minimum spanning tree of $T / K$, and $\operatorname{drop}_{T}(K)$ be its cost. We illustrate this in Figure 2. We state the Bridge Lemma here and present its proof for completeness.

Lemma 1 (Bridge Lemma [1]). Given a terminal spanning tree $T$ and a feasible solution $x$ to $(\mathcal{S})$,

$$
\sum_{K} x_{K} \mathrm{drop}_{T}(K) \geq c(T)
$$

Proof. The proof needs the following theorem of Edmonds [3]: given a graph $H=(R, F)$, the extreme points of the polytope

$$
\left\{z \in \mathbb{R}_{\geq 0}^{F}: \sum_{(u, v) \in F: u \in S, v \in S} z_{e} \leq|S|-1 \quad \forall S \subset R, \quad \sum_{e \in F} z_{e}=|R|-1\right\}
$$

are the indicator variables of spanning trees of $H$. The proof strategy is as follows. We construct a multigraph $H=(R, F)$ with $\operatorname{costs} c$, and $z \in \mathbb{R}^{F}$ such that: the cost of $z$ equals the left-hand side of $(1) ; z \in(\mathcal{G})$; and all spanning trees of $H$ have cost at least $c(T)$. Edmonds' theorem then immediately implies the lemma. In the rest of the proof we define $H$ and supply the three parts of this strategy.

For each full component $K$ with $x_{K}>0$, consider the edges in $\operatorname{Drop}_{T}(K)$. Contracting all edges of $E(T) \backslash \operatorname{Drop}_{T}(K)$, we see that $\operatorname{Drop}_{T}(K)$ corresponds to edges of a spanning tree of $K$. These edges are copied (with the same cost $c$ ) into the set $F$, and the copies are given weight $z_{e}=x_{K}$. Using the definition of drop, one can show each $e \in F$ is a maximum-cost edge in the unique cycle of $T \cup\{e\}$.

Having now defined $F$, we see

$$
\sum_{e \in F} c_{e} z_{e}=\sum_{K} x_{K} \mathrm{drop}_{T}(K)
$$

Note that we introduce $|K|-1$ edges for each full component $K$, and that, for any $S \subseteq R$, at most $|S \cap K|-1$ of these have both ends in $S$. These two observations together with the fact that $x$ is feasible for $(\mathcal{S})$ directly imply that $z$ is feasible for $(\mathcal{G})$.

To show all spanning trees of $H$ have cost at least $c(T)$, it suffices to show $T$ is an MST of $T \cup H$. In turn, this follows (e.g. [7, Theorem 50.9]) from the fact that each $e \in F$ is a maximum-cost edge in the unique cycle of $T \cup\{e\}$.

We also need two standard facts that we summarize in the following lemma. They rely on the input costs satisfying the triangle inequality, and that internal nodes of full components have degree at least 3 , both of which hold without loss of generality.

Lemma 2. (a) The value mst $(G[R])$ of the initial terminal spanning tree computed by algorithm RLC is at most twice the optimal value of $(\mathcal{S})$. (b) For any full component $K$, $\operatorname{loss}(K) \leq C_{K} / 2$.

Proof. See Lemma 4.1 in [4] for a proof of (b). For (a) we use a shortcutting argument along with Edmonds' polytope $(\mathcal{G})$ for the graph $H=G[R]$. In detail, let $x$ be an optimal solution to $(\mathcal{S})$. For each $K$, shortcut a tour of $\mathrm{F}(K)$ to obtain a spanning tree of $K$ with 
$c$-cost at most twice $C_{K}$ (by the triangle inequality) and add these edges to $F$ with $z$-value $x_{K}$. Like before, since $x$ is feasible for $(\mathcal{S}), z$ is feasible for $(\mathcal{G})$, and so there is a spanning tree of $G[R]$ whose $c$-cost is at most $\sum_{e \in F} c_{e} z_{e} \leq 2 \sum_{K} C_{K} x_{K}$.

We are ready to prove the main theorem.

Proof of Theorem 1. Let $x$ be an optimal solution to $(\mathcal{S})$ computed in step 2, define $1 \mathrm{p}^{*}$ to be its objective value, and

$$
\operatorname{loss}^{*}=\sum_{K} x_{K} \operatorname{loss}(K)
$$

its fractional loss. Our goal will be to derive upper bounds on the expected cost of tree $T_{i}$ maintained by the algorithm at the beginning of iteration $i$. After selecting $K_{i}$, one possible candidate spanning tree of $T_{i} \cup \operatorname{LC}\left(K_{i}\right)$ is given by the edges of $T_{i} \backslash \operatorname{Drop}_{T_{i}}\left(K_{i}\right) \cup \operatorname{LC}\left(K_{i}\right)$, and thus

$$
c\left(T_{i+1}\right) \leq c\left(T_{i}\right)-\operatorname{drop}_{T_{i}}\left(K_{i}\right)+c\left(\operatorname{LC}\left(K_{i}\right)\right) .
$$

Let us bound the expected value of $T_{i+1}$, given any fixed $T_{i}$. Due to the distribution from which $K_{i}$ is drawn, and using (3) with linearity of expectation, we have

$$
E\left[c\left(T_{i+1}\right)\right] \leq c\left(T_{i}\right)-\frac{1}{M} \sum_{K} x_{K} \operatorname{drop}_{T_{i}}(K)+\frac{1}{M} \sum_{K} x_{K}\left(C_{K}-\operatorname{loss}(K)\right) .
$$

Applying the bridge lemma on the terminal spanning tree $T_{i}$, and using the definitions of lp* and loss*, we have

$$
\mathbf{E}\left[c\left(T_{i+1}\right)\right] \leq\left(1-\frac{1}{M}\right) \mathbf{E}\left[c\left(T_{i}\right)\right]+\left(1 \mathrm{p}^{*}-1 \mathrm{oss}^{*}\right) / M
$$

By induction this gives

$$
\begin{aligned}
\mathbf{E}\left[c\left(T_{t+1}\right)\right] & =\left(1-\frac{1}{M}\right)^{t} c\left(T_{1}\right)+\left(1 \mathrm{p}^{*}-\operatorname{loss}^{*}\right)\left(1-\left(1-\frac{1}{M}\right)^{t}\right) \\
& \leq 1 \mathrm{p}^{*}\left(1+\left(1-\frac{1}{M}\right)^{t}\right)-\operatorname{loss}^{*}\left(1-\left(1-\frac{1}{M}\right)^{t}\right) .
\end{aligned}
$$

where the inequality uses Lemma 2(a). The cost of the final Steiner tree is at most $c(A L G) \leq$ $c\left(T_{t+1}\right)+\sum_{i=1}^{t} \operatorname{loss}\left(K_{i}\right)$. Moreover,

$$
\begin{aligned}
\mathbf{E}[c(A L G)] & \leq \mathbf{E}\left[c\left(T_{t+1}\right)\right]+t \cdot \operatorname{loss}^{*} / M \\
& \leq 1 \mathrm{p}^{*}\left(1+\left(1-\frac{1}{M}\right)^{t}\right)+\operatorname{loss}^{*}\left(\left(1-\frac{1}{M}\right)^{t}+\frac{t}{M}-1\right) \\
& \leq 1 \mathrm{p}^{*}\left(\frac{1}{2}+\frac{3}{2}\left(1-\frac{1}{M}\right)^{t}+\frac{t}{2 M}\right) \\
& \leq 1 \mathrm{p}^{*}(1 / 2+3 / 2 \cdot \exp (-t / M)+t / 2 M)
\end{aligned}
$$

where the third inequality uses (a weighted average of) Lemma 2(b). The last line explains our choice of $t=M \ln 3$ since $\lambda=\ln 3$ minimizes $\frac{1}{2}+\frac{3}{2} e^{-\lambda}+\frac{\lambda}{2}$, with value $1+\frac{\ln 3}{2}$. Thus the algorithm outputs a Steiner tree of expected cost at most $\left(1+\frac{\ln 3}{2}\right) l p^{*}$, which implies the claimed upper bound of $1+\frac{\ln 3}{2}$ on the integrality gap. 
We now discuss a variant of the result just proven. A Steiner tree instance is quasibipartite if there are no Steiner-Steiner edges. For quasibipartite instances, Robins and Zelikovsky tightened the analysis of their algorithm to show it has approximation ratio $\alpha$, where $\alpha \approx 1.28$ satisfies $\alpha=1+\exp (-\alpha)$ ). Here, we'll show an integrality gap bound of $\alpha$ (the longer proof of [1] via the Robins-Zelikovsky algorithm can be similarly adapted). We can refine Lemma 2(a) (like in [6]) to show that in quasi-bipartite instances, mst $(G[R]) \leq$ $2\left(1 \mathrm{p}^{*}-1 \mathrm{ss}^{*}\right)$. Continuing along the previous lines, we obtain

$$
\mathbf{E}[c(A L G)] \leq 1 \mathrm{p}^{*}(1+\exp (-t / M))+\operatorname{loss^{*}}(t / M-1-\exp (-t / M))
$$

and setting $t=\alpha M$ gives $\mathbf{E}[c(A L G)] \leq \alpha \cdot 1 \mathrm{p}^{*}$, as needed. We note that in quasi-bipartite instances the hypergraphic relaxation is equivalent [2] to the so-called bidirected cut relaxation thus we get an $\alpha$ integrality gap bound there as well.

At the risk of numerology, we conclude by remarking that $1+\frac{\ln 3}{2}$ arose in two very different ways, by analyzing different algorithms (and similarly for $\alpha \approx 1.28$ ). A simple explanation for this phenomenon would be very interesting.

\section{References}

[1] J. Byrka, F. Grandoni, T. Rothvoß, and L. Sanità. An improved LP-based approximation for Steiner tree. In Proc. 42nd STOC, pages 583-592, 2010.

[2] Deeparnab Chakrabarty, Jochen Könemann, and David Pritchard. Hypergraphic LP relaxations for Steiner trees. In Proc. 14th IPCO, pages 383-396, 2010. Full version at arXiv:0910.0281.

[3] J. Edmonds. Matroids and the greedy algorithm. Math. Programming, 1:127-136, 1971.

[4] C. Gröpl, S. Hougardy, T. Nierhoff, and H. J. Prömel. Approximation algorithms for the Steiner tree problem in graphs. In X. Cheng and D.Z. Du, editors, Steiner trees in industries, pages 235-279. Kluwer Academic Publishers, Norvell, Massachusetts, 2001.

[5] Tobias Polzin and Siavash Vahdati Daneshmand. On Steiner trees and minimum spanning trees in hypergraphs. Oper. Res. Lett., 31(1):12-20, 2003.

[6] G. Robins and A. Zelikovsky. Tighter bounds for graph Steiner tree approximation. SIAM J. Discrete Math., 19(1):122-134, 2005. Preliminary version appeared in Proc. 11th SODA, pages 770-779, 2000.

[7] A. Schrijver. Combinatorial optimization. Springer, New York, 2003.

[8] D.M. Warme. A new exact algorithm for rectilinear Steiner trees. In P.M. Pardalos and D.-Z. Du, editors, Network Design: Connectivity and Facilities Location, pages 357-395. American Mathematical Society, 1997. (Result therein attributed to M. Queyranne.). 